\title{
Analysis of gene co-expression network reveals prognostic significance of CNFN in patients with head and neck cancer
}

\author{
BAOLING LIU ${ }^{1-3}$, GUANHONG HUANG ${ }^{3}$, HONGMING ZHU², ZHAOMING MA ${ }^{3}$, \\ XIAOKANG TIAN ${ }^{2}, \mathrm{LI} \mathrm{YIN}^{2}, \mathrm{XINGYA} \mathrm{GAO}^{1^{*}}$ and $\mathrm{XIA} \mathrm{HE}^{2^{*}}$ \\ ${ }^{1}$ Department of Physiology, School of Basic Medical Sciences, Nanjing Medical University, Nanjing, Jiangsu 211166; \\ ${ }^{2}$ Department of Radiotherapy, Jiangsu Cancer Hospital, Jiangsu Institute of Cancer Research, \\ Nanjing Medical University Affiliated Cancer Hospital, Nanjing, Jiangsu 210000; ${ }^{3}$ Department of Radiotherapy, \\ No. 2 People's Hospital of Lianyungang, Lianyungang, Jiangsu 222000, P.R. China
}

Received August 20, 2018; Accepted February 7, 2019

DOI: $10.3892 /$ or.2019.7019

\begin{abstract}
In patients with head and neck cancer (HNC), lymph node $(\mathrm{N})$ metastases are associated with cancer aggressiveness and poor prognosis. Identifying meaningful gene modules and representative biomarkers relevant to the $\mathrm{N}$ stage helps predict prognosis and reveal mechanisms underlying tumor progression. The present study used a step-wise approach for weighted gene co-expression network analysis (WGCNA). Dataset GSE65858 was subjected to WGCNA. RNA sequencing data of HNC downloaded from the Cancer Genome Atlas (TCGA) and dataset GSE39366 were utilized to validate the results. Following data preprocessing, 4,295 genes were screened, and blue and black modules associated with the $\mathrm{N}$ stage of HNC were identified. A total of 16 genes [keratinocyte differentiation associated protein, suprabasin, cornifelin (CNFN), small proline rich protein $1 \mathrm{~B}$, desmoglein 1 (DSG1), chromosome 10
\end{abstract}

Correspondence to: Dr Xingya Gao, Department of Physiology, School of Basic Medical Sciences, Nanjing Medical University, 101 Longmian Avenue, Jiangning, Nanjing, Jiangsu 211166, P.R. China E-mail: gaoxy@njmu.edu.cn

Dr Xia He, Department of Radiotherapy, Jiangsu Cancer Hospital, Jiangsu Institute of Cancer Research, Nanjing Medical University Affiliated Cancer Hospital, 42 Bai Zi Ting Road, Nanjing, Jiangsu 210000, P.R. China

E-mail: hexiabm@163.com

*Contributed equally

Abbreviations: HNC, head and neck cancer; T, primary tumor; $\mathrm{N}$, lymph node; WGCNA, weighted gene co-expression network analysis; TCGA, The Cancer Genome Atlas; GEO, Gene Expression Omnibus; GEPIA, Gene Expression Profiling Interactive Analysis; GSEA, gene set enrichment analysis; TOM, topological overlap matrix; MEs, module eigengenes; GS, Gene significance; MS, module significance

Key words: HNC, cornifelin, co-expression, bioinformatics analysis, prognosis open reading frame 99 , keratin 16 pseudogene 3 , gap junction protein $\beta 2$, dermokine, LY6/PLAUR domain containing 3, transmembrane protein 79, phospholipase A2 group IVE, transglutaminase 5 , potassium two pore domain channel subfamily $\mathrm{K}$ member 6 , involucrin, kallikrein related peptidase 8] that had a negative association with the $\mathrm{N}$-stage in the blue module, and two genes (structural maintenance of chromosomes 4 and mutS homolog 6) that had a positive association in the black module, were identified to be candidate hub genes. Following further validation in TCGA and dataset GSE65858, it was identified that CNFN and DSG1 were associated with the clinical stage of HNC. Survival analysis of CNFN and DSG1 was subsequently performed. Patients with increased expression of CNFN displayed better survival probability in dataset GSE65858 and TCGA. Therefore, CNFN was selected as the hub gene for further verification in the Gene Expression Profiling Interactive Analysis database. Finally, functional enrichment and gene set enrichment analyses were performed using datasets GSE65858 and GSE39366. Three gene sets, namely 'P53 pathway', 'estrogen response early' and 'estrogen response late', were enriched in the two datasets. In conclusion, CNFN, identified via the WGCNA algorithm, may contribute to the prediction of lymph node metastases and prognosis, probably by regulating the pathways associated with P53, and the early and late estrogen response.

\section{Introduction}

Head and neck cancer (HNC) has a mortality rate of $40-50 \%$ and is detected in 600,000 cases annually worldwide, accounting for $\sim 3.8 \%$ of global cancer cases and $\sim 3.6 \%$ of all cancer-associated mortality $(1,2)$. The American Joint Committee on Cancer staging system for HNC demands an integrated assessment of the patient, primarily including the primary tumor $(\mathrm{T})$, lymph node metastasis $(\mathrm{N})$ and distant metastasis (3). Traditionally, prognosis has been associated with tumor stage (4,5). Pereira et al (6) emphasized that lymph node metastases ( $\mathrm{N}$ stage) in patients with cancer are associated with tumor aggressiveness, recommendation for systemic therapy and poor prognosis. Different etiologies and a large variety of molecular alterations drive HNC to be a markedly 
heterogeneous disease. Recognizing the prognostic power of lymph node metastases, the identification of potential novel biomarkers associated with the lymph node stage of $\mathrm{HNC}$ is thus meaningful.

The understanding of human diseases ultimately depends on the understanding of the genome and its functions (7). The recent application of microarray and sequencing technologies to transcriptomics has altered the view of cancer diagnosis, treatment and prognostic speculation. Over the past few years, subgroups of HNC characterized by gene expression patterns have been identified using expression arrays and RNA sequencing (8-10). Tartour et al (11) revealed that serum sIL-2R $\alpha$ may be considered as an independent serum biomarker in patients with HNC. Lin et al (12) reported that C1GALT1 serves a critical role in HNC progression and highlighted the therapeutic potential of targeting this gene during $\mathrm{HNC}$ treatment. Rettig et al (13) identified that HEY1 is expressed independently of NOTCH1 and is associated with a poor prognosis in HNC. Nevertheless, the majority of existing studies are limited to screening for genes with differential expression, and ignore the close connections between them.

Weighted gene co-expression network analysis (WGCNA) is systematic bioinformatics approach used to describe the associations among genes across microarray samples (14). This method may be used to find modules of tightly correlated genes, summarize these modules using an intramodular hub gene or the module eigengene and calculate module membership measures. At present, it has been generally acknowledged and used to identify hub genes in various cancer types, including breast cancer (15), pancreatic carcinoma (16) and osteosarcoma (17). By constructing co-expression networks, 10 hub genes in oral squamous cell carcinoma were identified and validated (18). $\mathrm{Li}$ (19) reported that TPX2, microtubule nucleation factor (TPX2), minichromosome maintenance complex component 2, ubiquitin like with PHD and ring finger domains 1 , cyclin dependent kinase 2 and protein regulator of cytokinesis 1 were associated with the tumorigenesis of laryngeal squamous cell carcinoma. However, previous studies have primarily identified hub genes associated with the pathogenesis of cancer, and studies associated with prognosis have not been reported. In the present study, a co-expression network of interconnection between the genes of HNC was constructed using WGCNA analysis, and network-centric genes associated with tumor prognosis were identified.

\section{Materials and methods}

Study design. To clarify the data collection, preprocessing, analysis and validation, a schematic of the research process is presented in Fig. 1.

Data collection. Raw mRNA expression profiles of HNC were downloaded from the Gene Expression Omnibus (GEO) database (http://www.ncbi.nlm.nih.gov/geo/), a public data repository of functional genomics data. Using the search terms 'head and cancer [MeSH Terms] AND Expression profiling by array [DataSet Type] AND Homo sapiens [Organism]' in the GEO datasets, datasets GSE65858 and GSE39366 were further screened. Dataset GSE65858 performed on Illumina HumanHT-12 V4.0 Expression Beadchips (Illumina Inc.,
San Diego, CA, USA), including 270 head and neck squamous cell carcinoma (HNSC) tumor samples with clinical and prognostic variables (20), was used for constructing a weighted gene co-expression network and subsequently for identifying hub genes. As a validation set, dataset GSE39366 performed on Agilent-UNC-custom-4X44K (Agilent Technologies, Inc., Santa Clara, CA, USA), consisting of 138 HNSC samples, was used to verify the results (9). Moreover, RNA sequencing data for HNC were downloaded from the Cancer Genome Atlas (TCGA) database (https://portal.gdc.cancer.gov/repository), consisting of 500 tumor samples with complete expression profiles and clinical information and 44 normal tissues, to further validate the results.

Data preprocessing and screening. With regard to dataset GSE65858, robust multiarray averaging background correction was performed with the raw expression data, and the processed signals were subjected to $\log 2$ transformation and quantile normalization. The 'affy' R package (21) was used to summarize the median polished probe sets. According to the distances between different samples in average linkage, microarray quality was assessed via sample clustering, and no samples from GSE65858 were removed from the subsequent analysis. The standard deviation values for gene expression were obtained from the expression matrices. Subsequently, the genes were ranked and the top $25 \%$ were screened for the following analysis.

Weighted gene co-expression network construction. Given that gene co-expression analysis is extremely sensitive to the existence of abnormal samples, strict quality control procedures were implemented to ensure the highest quality level, followed by step-by-step network construction and module detection. To construct a scale-free gene co-expression network, the WGCNA package in R $(14,22,23)$ was used. First, Pearson's correlation matrices were performed on all gene pairs. Next, using the power function $a m n=|c m n| \beta$ (where amn is the adjacency between genes $\mathrm{m}$ and $\mathrm{n}$, and $\mathrm{cmn}$ is the Pearson's correlation between genes $m$ and $n$ ), a weighted adjacency matrix was constructed. As a soft-thresholding parameter, parameter $\beta$ may penalize weak correlations between genes while emphasizing strong correlations. To ensure a scale-free network in the present study, the power of $\beta=4$ (scale free $R^{2}=0.91$ ) was selected (24). Then, the adjacency was transformed into a topological overlap matrix (TOM); TOM is defined as the contiguous sum with all the other genes used for network generation and for measurement of the network connectivity of genes (25). Then, we calculated the corresponding dissimilarity (1-TOM). To classify genes with similar expression profiles into different modules, average linkage hierarchical clustering was performed, according to TOM-based dissimilarity measures; the minimum size (genome) of the gene dendrogram was 50 (26). To investigate the module further, the dissimilarity of module eigengene (MEs) was calculated, a cut line for the module dendrogram was selected, and certain modules were merged (16).

Clinically significant modules and hub gene identification. When the initial set of modules had been created, the correlations among MEs were used to merge close modules. MEs, 


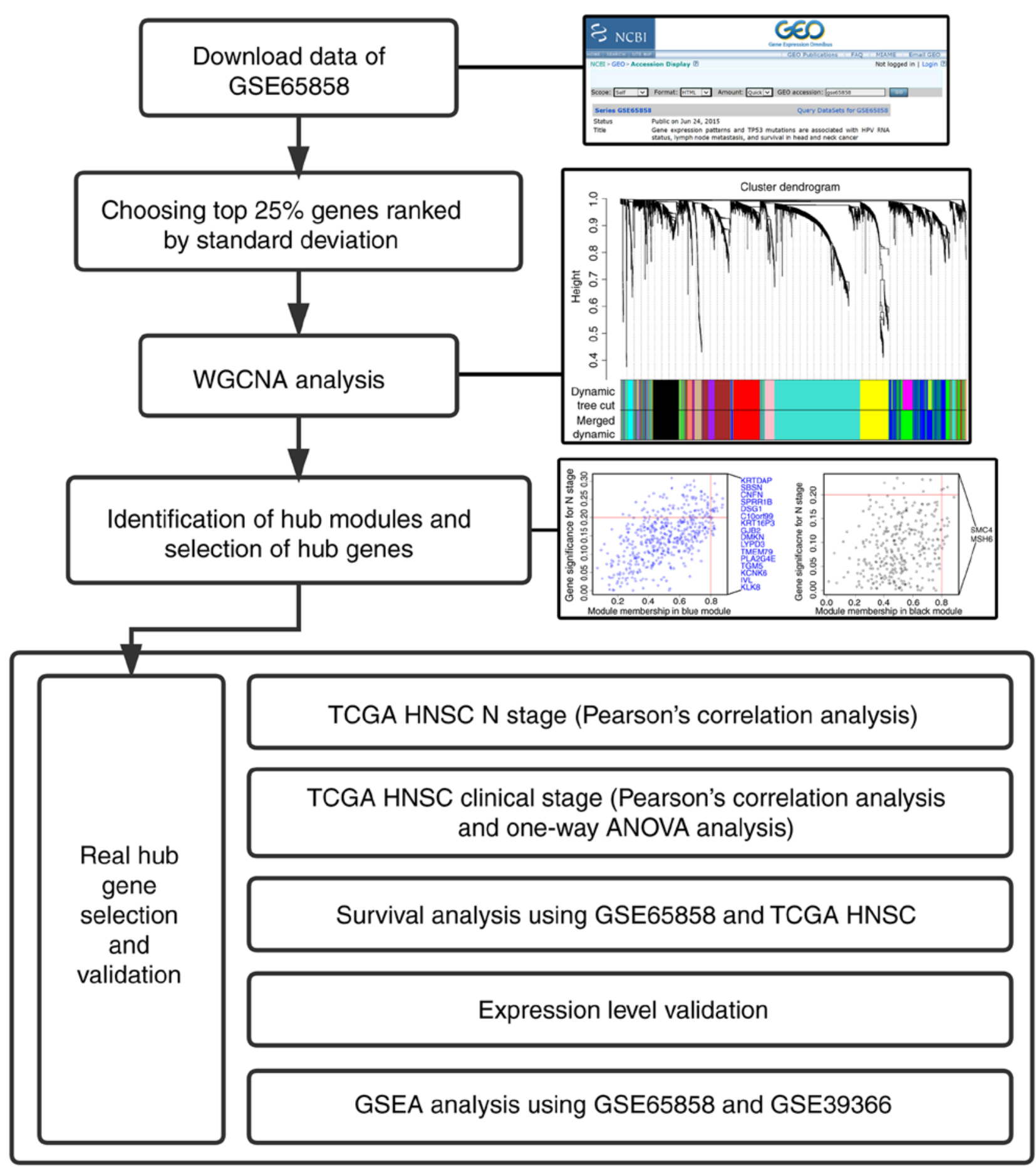

Figure 1. Flow diagram of the study design, illustrating data preparation, preprocessing, analysis and validation. ANOVA, analysis of variance; WGCNA, weighted gene co-expression network analysis; TCGA, The Cancer Genome Atlas; N, lymph node.

the first major component of gene expression within a module, summarize the feature expression patterns of modules, and modules with extremely similar expression profiles display highly correlated eigengenes (27). Gene significance (GS) refers to the $\log 10$ conversion of the $\mathrm{P}$-value in a linear regression $(\mathrm{GS}=\lg \mathrm{P})$ between clinical traits and gene expression, and module significance (MS) refers to the average GS of all genes in the module.

Typically, modules with an absolute MS ranking first or second in all modules are considered candidates relevant to clinical traits (16).
It has been demonstrated that hub genes, defined as genes that are strongly connected with others in a module, have a significant function (16). In this study, upon selecting modules of interest, the hub genes by the conditions of module connectivity (cor.geneModuleMembership $>0.8$ ) and clinical trait relationship (cor.geneTraitSignificance $>0.2$ ), which were measured by the absolute Pearson's correlation value (15). To identify key hub genes among the candidates, a linear regression analysis was performed to assess the link between the clinical features of interest and the expression of hub genes, and $\mathrm{R}^{2}$ was defined as the association between them. 


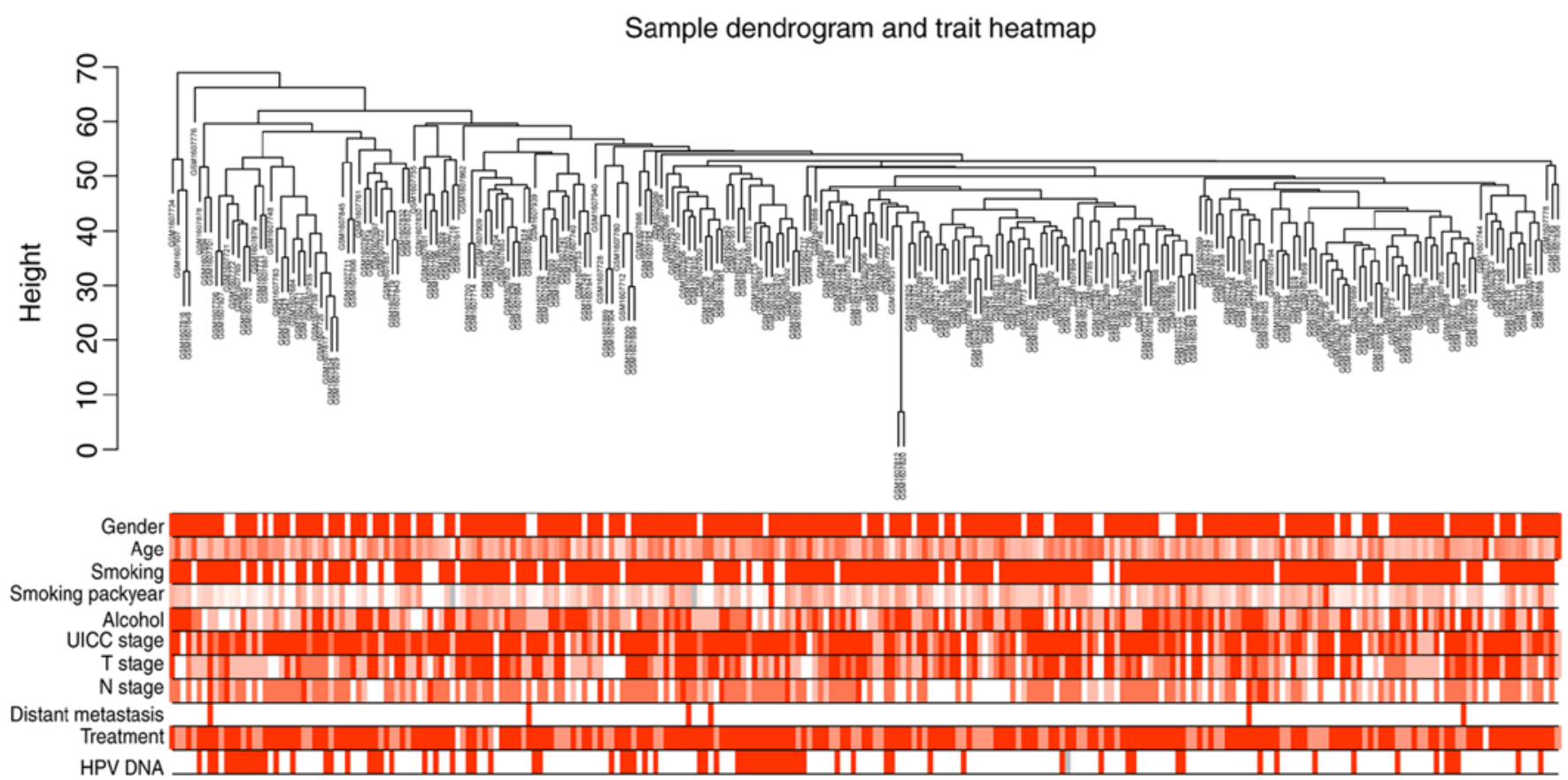

Figure 2. Sample cluster dendrogram and trait indicators. T, primary tumor; N, lymph node; UICC, Union for International Cancer Control; HPV, human papillomavirus.

Real hub gene validation. The training set (GSE65858), test set TCGA HNSC and public database Gene Expression Profiling Interactive Analysis (GEPIA) were used to identify the real hub genes. First, a Pearson correlation analysis of the $\mathrm{N}$-stage gene expression was performed using the GSE65858 and TCGA HNSC datasets. Pearson correlation analysis of clinical staging gene expression and one-way analysis of variance (ANOVA) were conducted using the GSE65858 and TCGA HNSC datasets. Subsequently, survival analysis for these genes was performed using the GSE65858 and TCGA HNSC datasets. Genes in all tests with significant P-values were identified as true hub genes. To verify the results further, the GEPIA database (http://www.gepia.cancer-pku.cn) was used to validate the expression levels of the real hub genes.

Gene set enrichment analysis (GSEA). The samples in the GSE65858 and GSE39366 datasets were respectively divided into two groups based on the median expression levels of the real hub genes. To further analyze the potential function of the hub genes further, GSEA analysis (http://software.broadinstitute.org/gsea/index.jsp) was performed to detect whether genes in the two groups were enriched with meaningful biological processes (28). The annotated gene set collection sh.all.v6.1.symbols.gmt [Hallmarks] in the molecular signatures database (MSigDB; http://software.broadinstitute. org/gsea/msigdb/index.jsp) was selected as the reference. Furthermore, $\mathrm{P}<0.05$ was set as the cut-off criterion. In addition, a Venn plot was generated based on the results for the GSE65858 and GSE39366 datasets.

\section{Results}

Training set quality assessment and gene screening. As indicated in the workflow in Fig. 1, the gene expression matrices from the 270 samples in training set GSE65858 were first downloaded following data preprocessing. The standard deviation values of gene expression were obtained from the expression matrices. The genes were ranked and the top $25 \%(4,295$ genes) were screened for subsequent analysis.

WGCNA identifies key modules. Following the initial quality assessment performed using the WGCNA R package via the average linkage method, no samples were removed from the GSE65858 dataset for the subsequent analysis (Fig. 2). As presented in Fig. 2, a total of 11 clinical traits had been identified, including gender, age, smoking, smoking pack years, alcohol, UICC stage, T stage, $\mathrm{N}$ stage, distant metastasis, treatment and HPV DNA status. To ensure a scale-free network, the scale-free fit index and mean connectivity were calculated and the power of $\beta=4$ (scale free $R^{2}=0.91$ ) was selected to perform further analysis (Fig. 3). Moreover, genes with similar expression patterns could be placed into different modules via average linkage clustering. Finally, 12 modules were identified (Fig. 4). Two methods, namely module-trait relationship and MS analysis, were used to examine the associations between the clinical traits and each module (Fig. 5A and B). First, modules with a better MS were considered to have a closer association with meaningful clinical traits of interest. It was identified that two modules, namely the blue and black modules, had higher MS values compared with the other modules. They were identified as the modules most relevant to the $\mathrm{N}$ stage of $\mathrm{HNC}$.

Candidate hub gene identification. Based on module connectivity, clinical trait relationship, and absolute value of Pearson's correlation (cor.geneModuleMembership $>0.8$, cor.geneTraitSignificance $>0.2$ ), we selected 18 genes with 
A

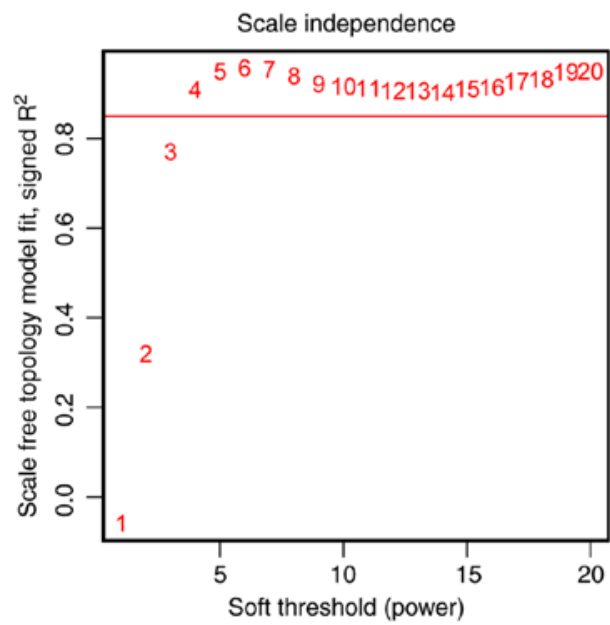

C

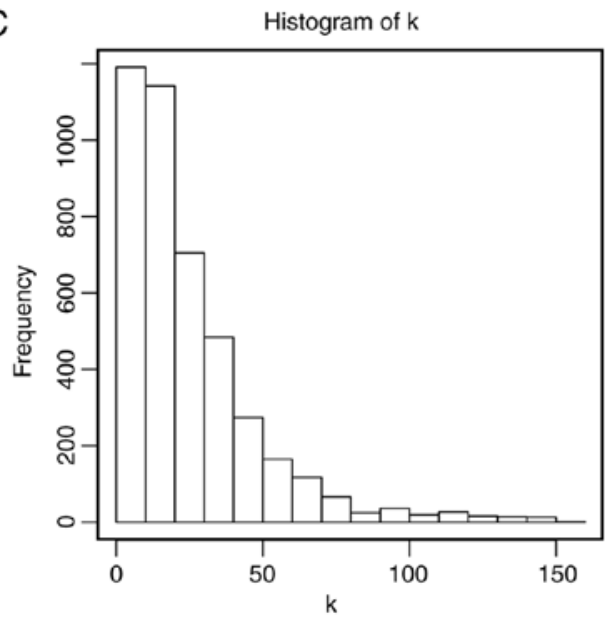

B

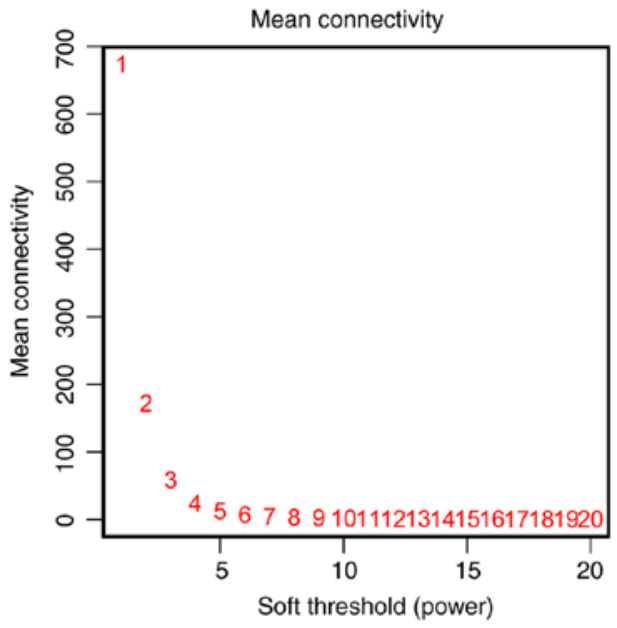

D

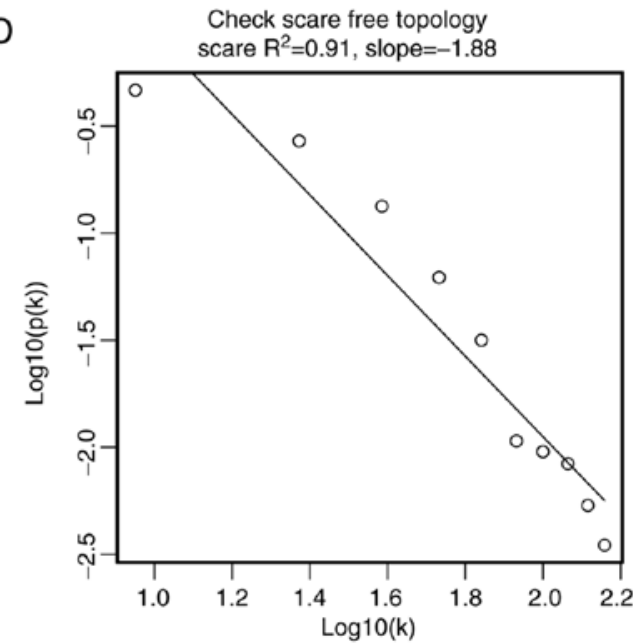

Figure 3. Determination of soft-thresholding power in the weighted gene co-expression network analysis. (A) Analysis of the scale-free fit index for various soft-thresholding powers ( $\beta$ ). (B) Analysis of the mean connectivity for various soft-thresholding powers. (C) Histogram of connectivity distribution when $\beta=4$. (D) Checking the scale-free topology when $\beta=4$.

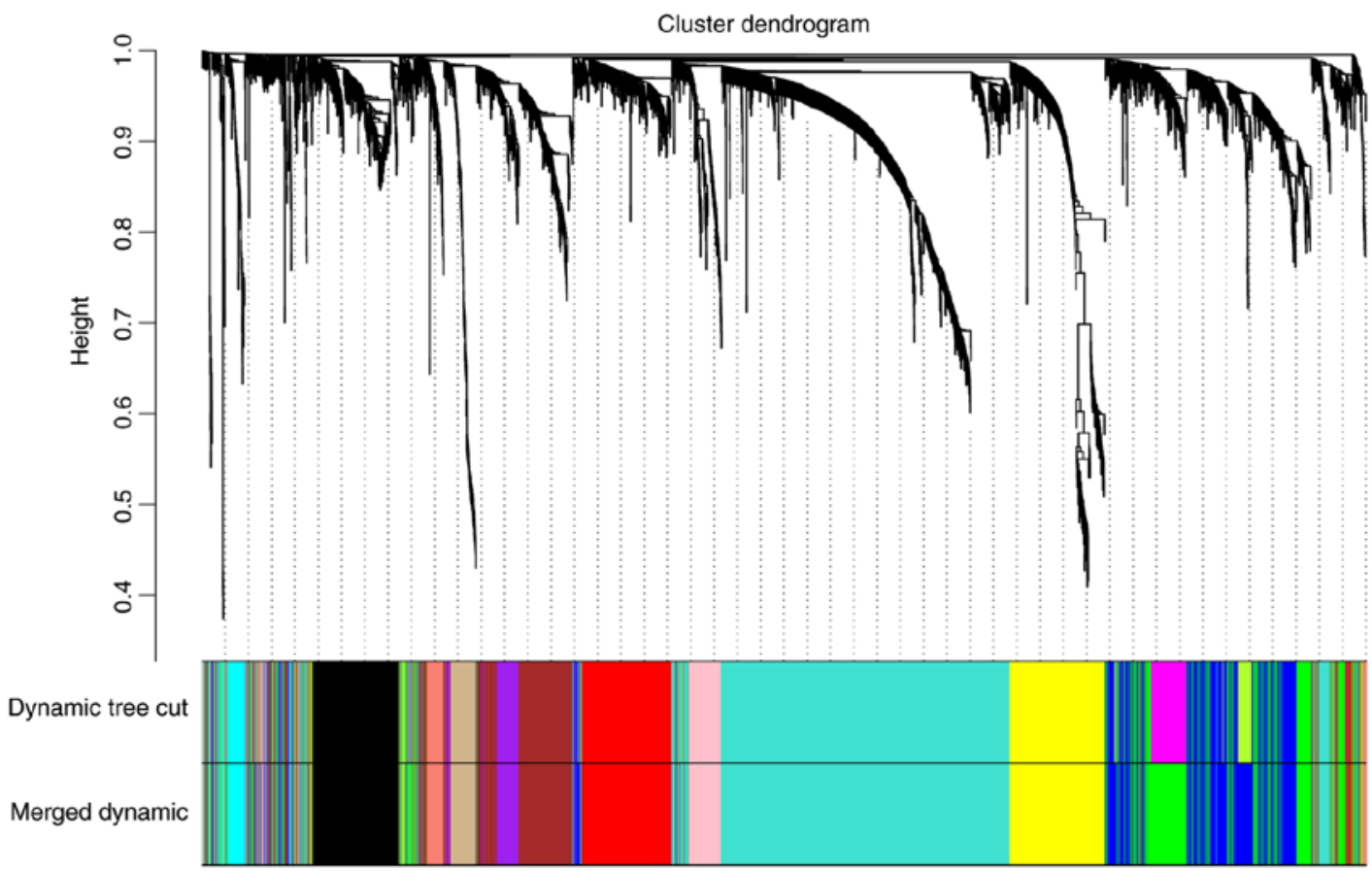

Figure 4. Dynamic tree cut. Dendrogram of all differentially expressed genes, which are clustered based on a dissimilarity measure (1-topological overlap matrix). 
A

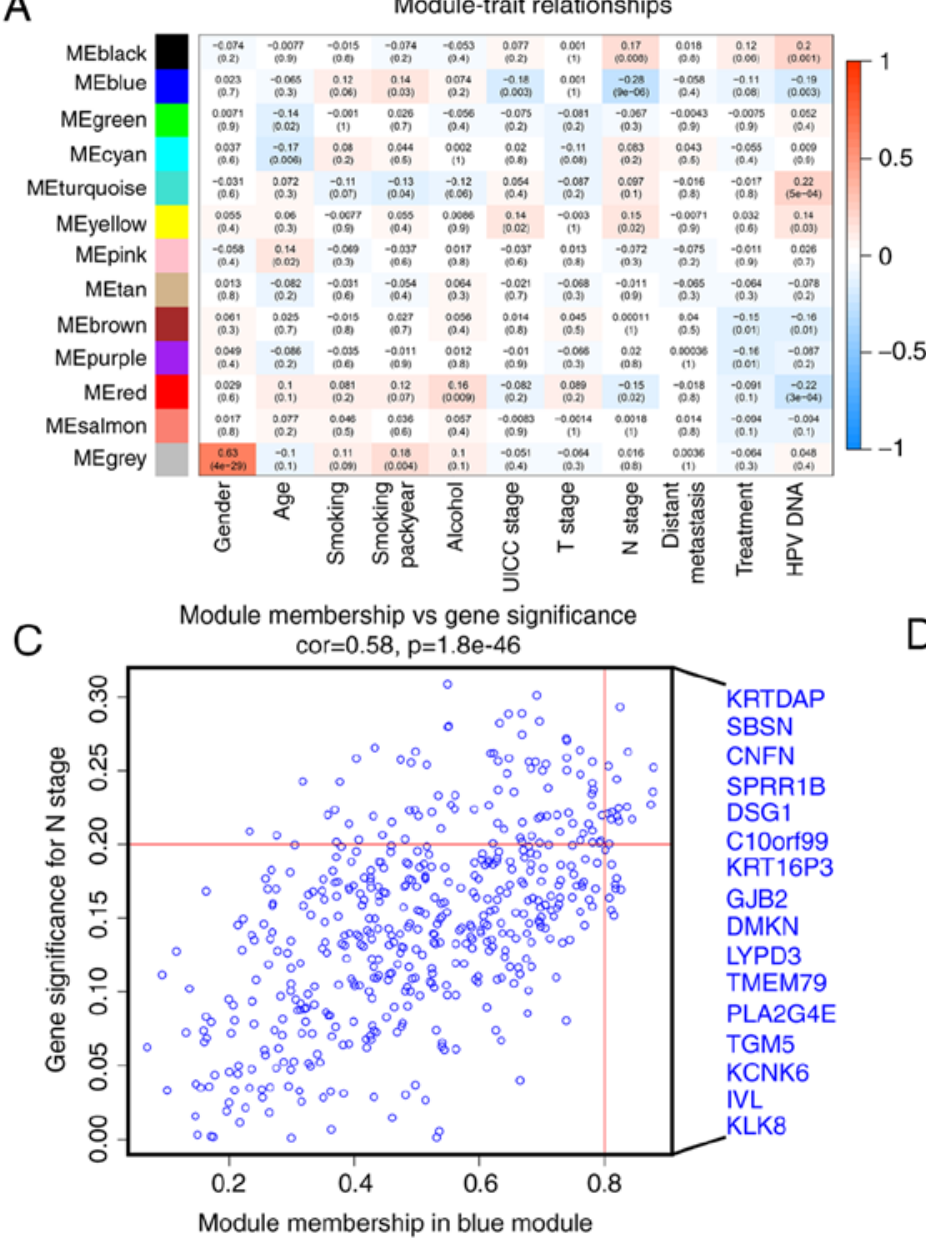

B

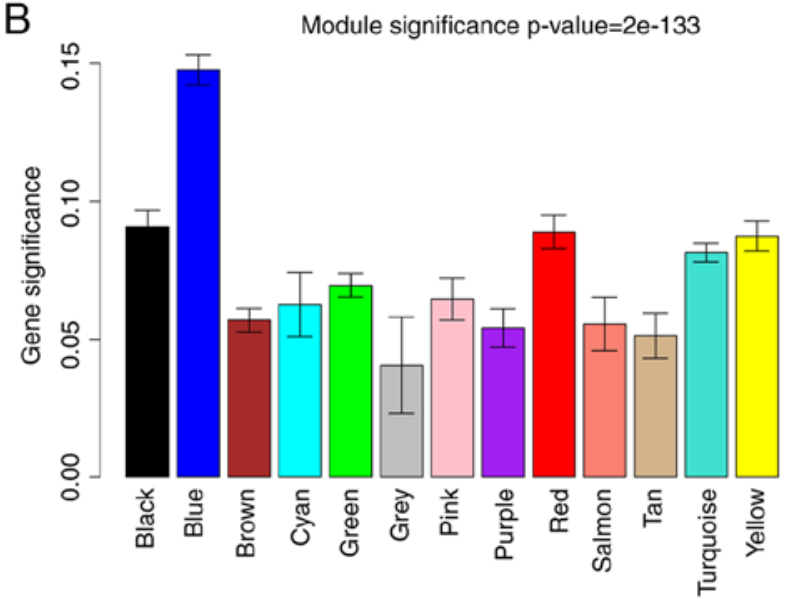

D

Figure 5. Identification of modules associated with clinical information. (A) Heatmap of the correlation between ME and different clinical information of HNSC (gender, age, smoking, smoking pack years, alcohol, UICC Stage, T stage, N stage, distant metastasis, treatment and HPV DNA). (B) Distribution of average gene significance and errors in the modules associated with the N stage of HNSC. Scatter plot of module eigengenes in the (C) blue and (D) black modules. T, primary tumor; N, lymph node; UICC, Union for International Cancer Control; HPV, human papillomavirus; ME, module eigengene; HNSC, head and neck squamous cell carcinoma.

tight connectivity as candidate hub genes in the two modules (Fig. 5C and D). A total of 16 genes, keratinocyte differentiation associated protein, suprabasin, cornifelin (CNFN), small proline rich protein 1B (SPRR1B), desmoglein 1 (DSG1), chromosome 10 open reading frame 99 (C10orf99), keratin 16 pseudogene 3, gap junction protein $\beta 2$ (GJB2), dermokine, LY6/PLAUR domain containing 3 (LYPD3), transmembrane protein 79, phospholipase A2 group IVE (PLA2G4E), transglutaminase 5 (TGM5), potassium two pore domain channel subfamily K member 6, involucrin (IVL) and kallikrein related peptidase 8 (KLK8), which were negatively correlated with the $\mathrm{N}$-stage, were identified as candidates in the blue module. Meanwhile, structural maintenance of chromosomes 4 and mutS homolog 6 (MSH6) were identified as candidates in the black module with a positive association.

Real hub gene identification and validation. To further validate the hub genes in TCGA, the candidate hub gene expression $\mathrm{N}$ stage mRNA sequencing data of 500 patients with HNC were subjected to Pearson's correlation analysis (Table I). Genes with significant differences in the two networks (C10orf99, CNFN, DSG1, GJB2, IVL, KLK8, LYPD3, MSH6, PLA2G4E,
SPRR1B and TGM5) were selected as the hub genes for further analysis and validation (Fig. 6). N stage is associated with clinical stage; to validate this correlation further, the GSE65858 dataset containing $270 \mathrm{HNC}$ tumors and mRNA sequencing data with clinical and prognostic variables of patients with HNC in TCGA were separately subjected to Pearson's correlation analysis and one-way ANOVA (Table I). Among all genes selected as candidate hub genes, only CNFN and DSG1 were found in the two networks (Fig. 7). Considering that the progression of a tumor affects patient prognosis, a survival analysis of CNFN and DSG1 was performed. Furthermore, it was observed that patients with increased CNFN expression had an improved survival probability in the GSE65858 and TCGA datasets (Fig. 8A and B), compared with DSG1, which only exhibited its prognostic role in the TCGA HNSC dataset. Therefore, CNFN was identified as the real hub gene for further validation. In addition, it was identified that in the GEPIA database, the specific expression of CNFN in normal samples of HNSC was significantly higher than other tumors and other normal tissues. More convincingly, the expression of CNFN exhibited a significant downregulation in HNSC tissues compared with normal samples (Fig. 8C and D). 


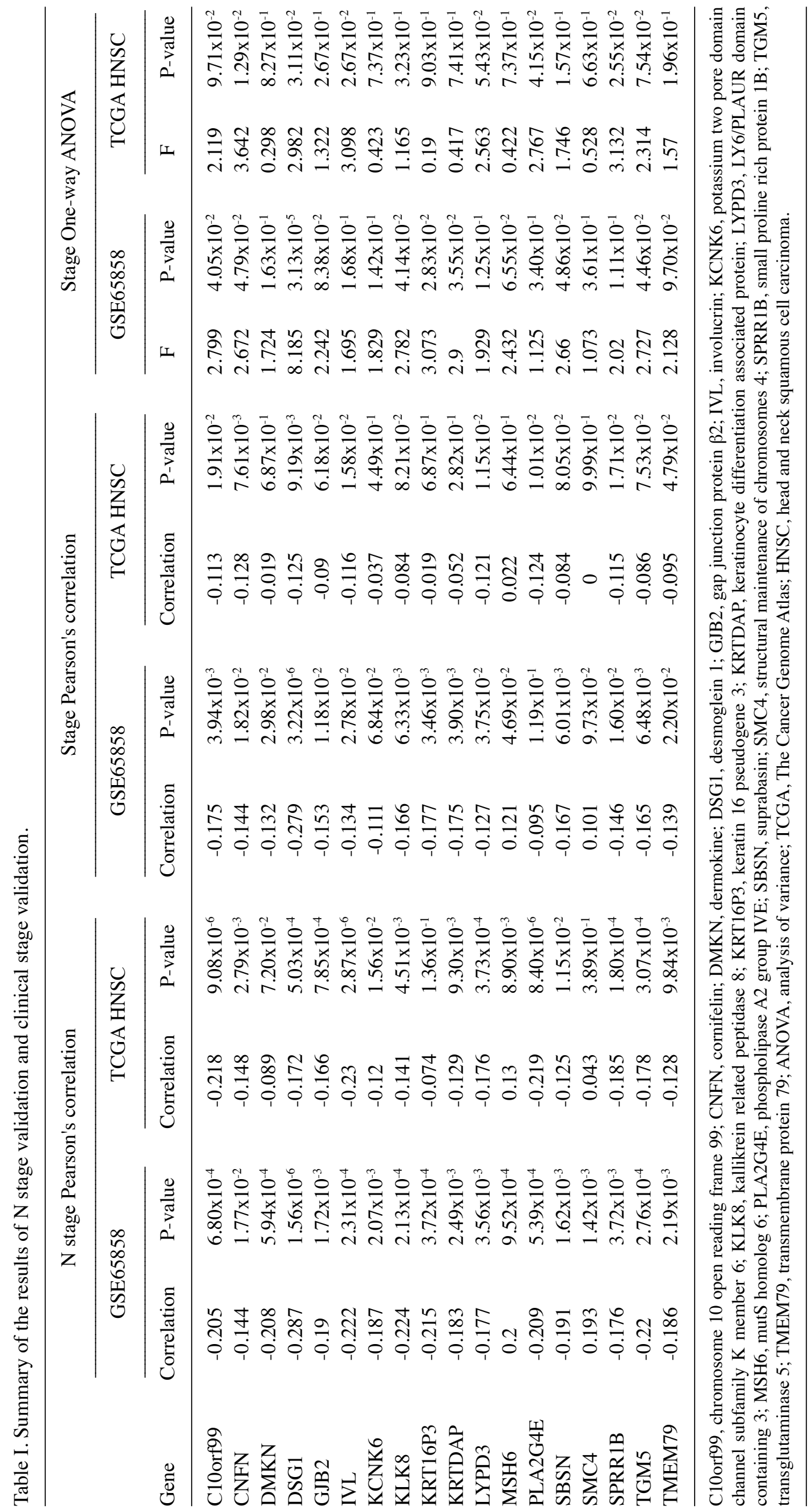


A
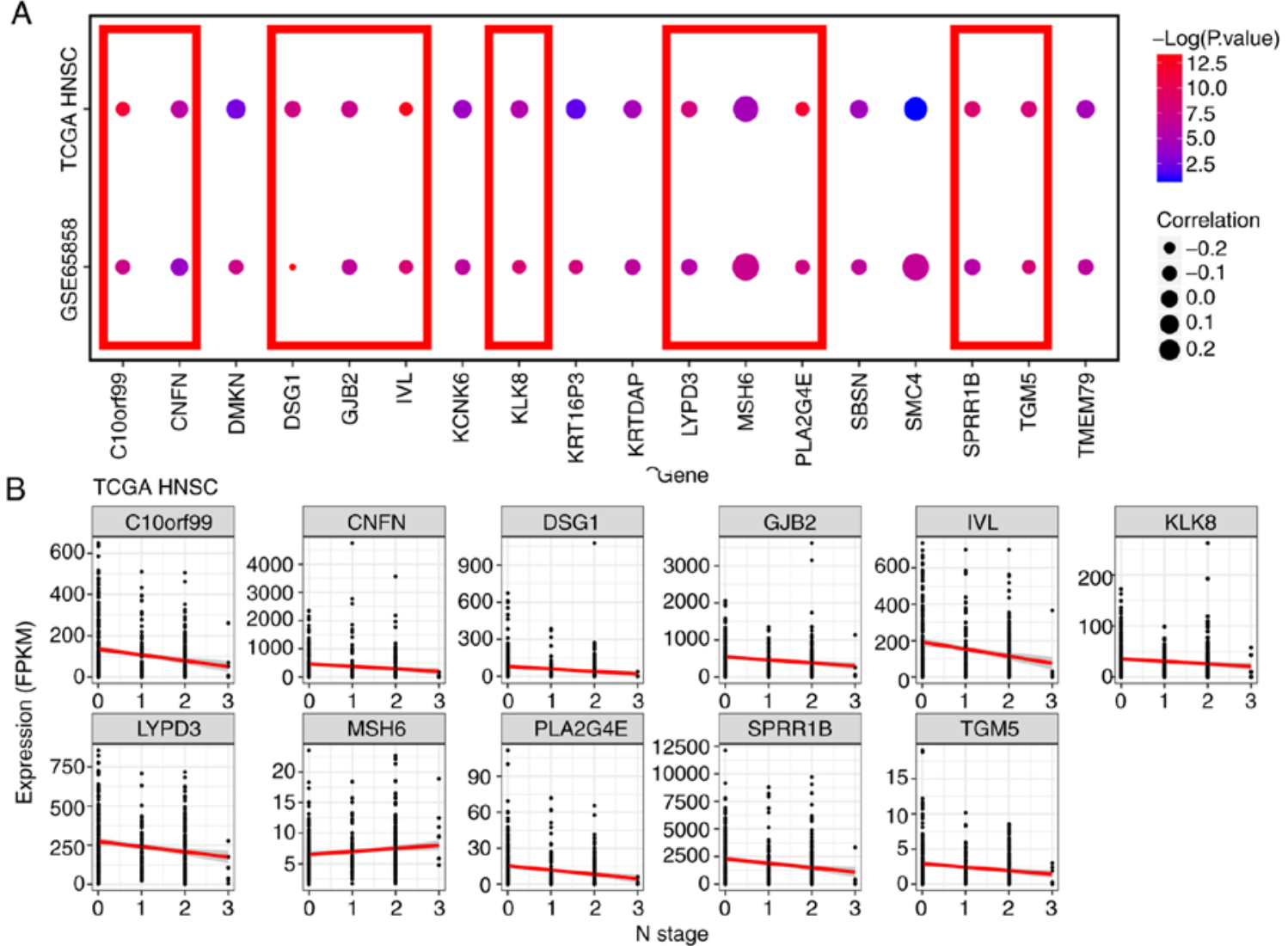

Figure 6. N stage validation. (A) Bubble plot of N stage correlation in the GSE65858 and TCGA HNSC datasets. (B) Genes with a significant P-value of N stage correlation. TCGA, The Cancer Genome Atlas; FPKM, fragments per kilobase of transcript per million mapped reads; HNSC, head and neck squamous cell carcinoma; N, lymph node.

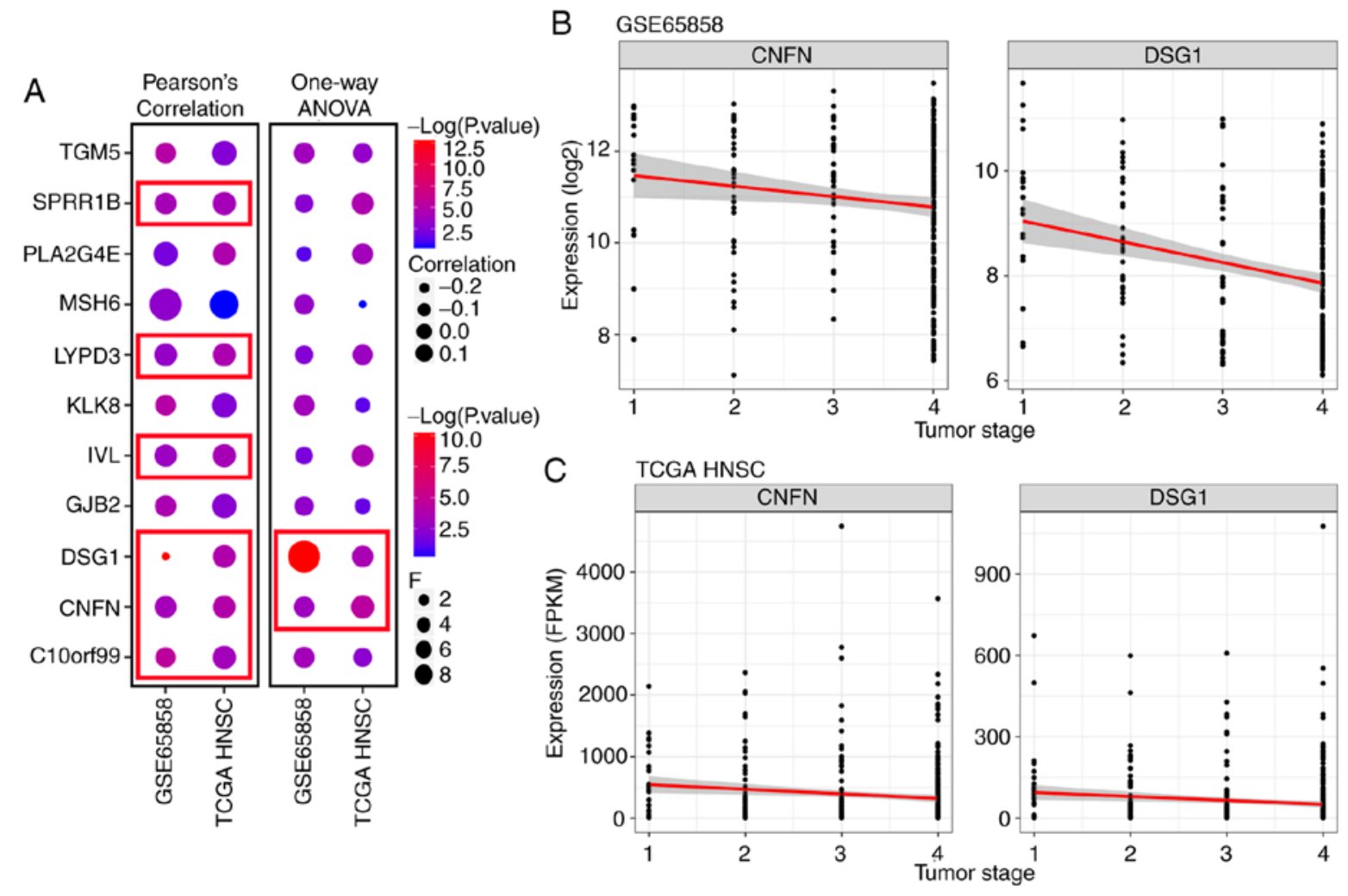

Figure 7. Clinical stage validation. (A) Bubble plot of stage correlation and one-way ANOVA in the GSE65858 and TCGA HNSC datasets. Genes with significant P-values of stage correlation and one-way ANOVA in the (B) GSE65858 and (C) TGCA HNSC datasets. ANOVA, analysis of variance; TCGA, The Cancer Genome Atlas; HNSC, head and neck squamous cell carcinoma; FPKM, fragments per kilobase of transcript per million mapped reads. 
A

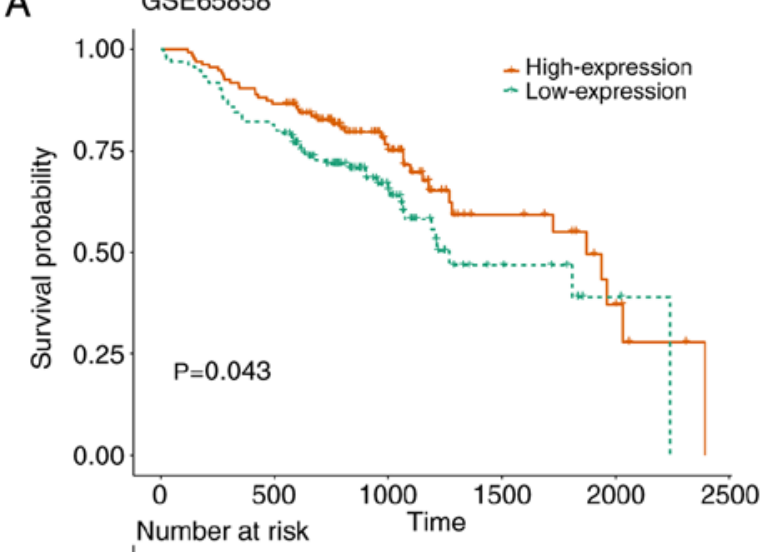

B

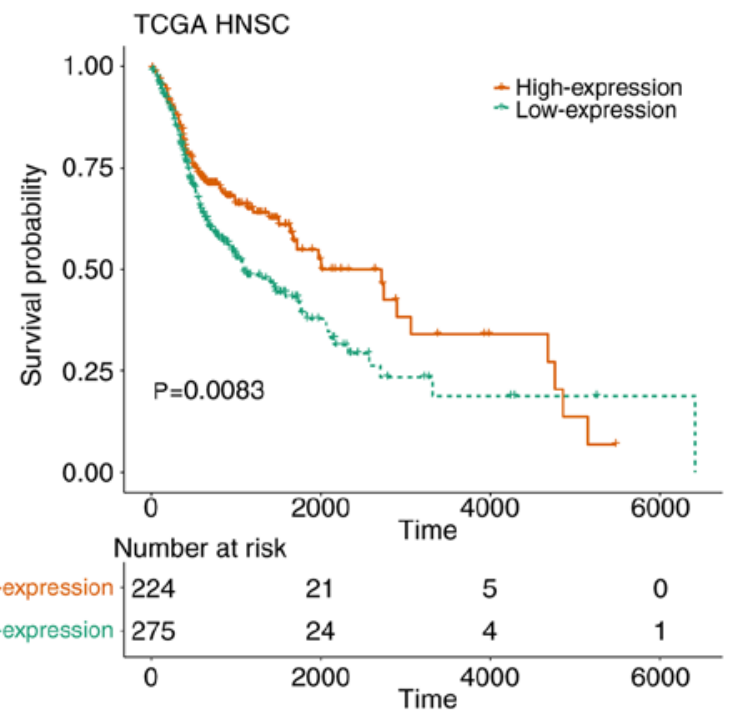

C

Low-expression $\begin{array}{cccccc}135 & 117 & 51 & 16 & 6 & 0 \\ & 109 & 46 & 9 & 2 & 0 \\ 0 & 500 & \begin{array}{c}1000 \\ \text { Time }\end{array} & 1500 & 2000 & 2500\end{array}$
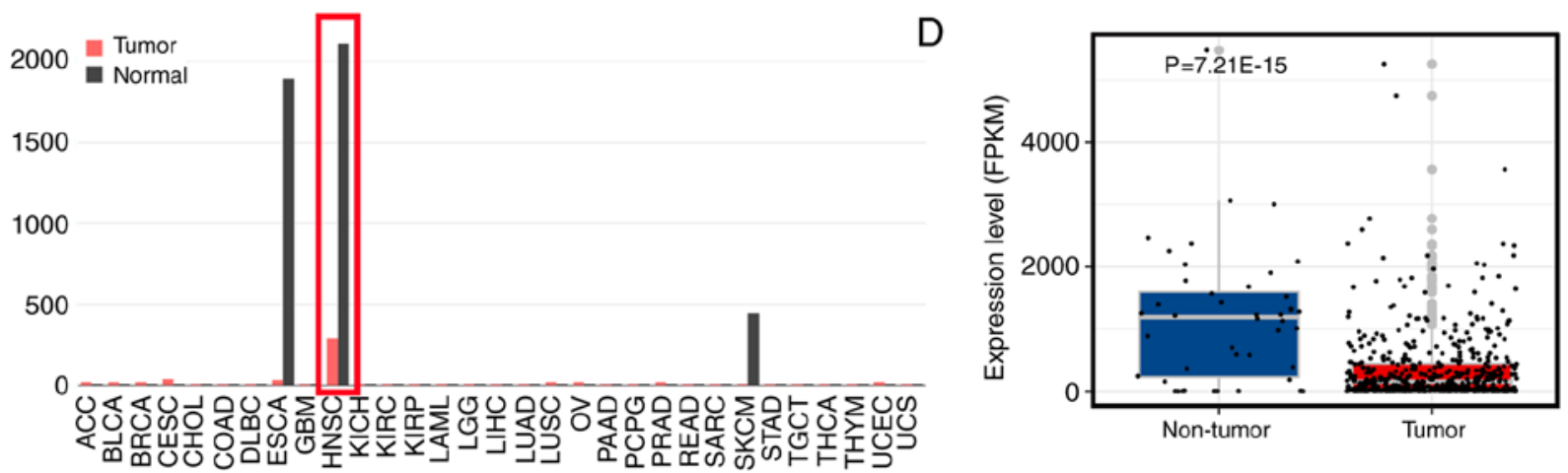

Figure 8. Outcome and expression level validation. (A) Overall survival analysis using GSE65858. (B) Overall survival analysis using TCGA HNSC. (C) CNFN expression level in all tumors and normal tissues using the Gene Expression Profiling Interactive Analysis database. (D) Expression level of CNFN using TCGA HNSC. TCGA, The Cancer Genome Atlas; HNSC, head and neck squamous cell carcinoma; CNFN, cornifelin; FPKM, fragments per kilobase of transcript per million mapped reads.

Gene set enrichment analysis. Kyoto Encyclopedia of Genes and Genomes pathway enrichment analysis is only used for the analysis of differentially expressed genes (DEGs), whereas GSEA analysis uses all probes or genes in the microarray, regardless of whether the gene is a DEG or not (15). GSEA analysis was performed in the present study using the GSE65858 and GSE39366 datasets. A total of 10 gene sets were enriched in GSE65858, while three were enriched in GSE39366. A total of three gene sets, 'P53 pathway', 'estrogen response early' and 'estrogen response late', were enriched in both datasets (Fig. 9).

\section{Discussion}

Head and neck oncology encompasses a group of malignancies that arise in the mucosal surfaces of the upper aerodigestive tract, including the oral cavity, pharynx, larynx and paranasal sinuses, in addition to cancer of the major and minor salivary glands (29). In addition, squamous cell carcinomas are the most common head and neck malignancies. Assigning the proper clinical stage, estimating prognosis and planning treatment are key for clinicians treating patients with cancer (29). Solid tumor progression is characterized by regional lymph nodes metastasis and distant organ dissemination. A number of studies have demonstrated that the presence of lymph node metastasis in cancer patients is correlated with a poor prognosis and determines the course of treatment to a certain extent (30-32). Further studies are required with respect to lymph node metastasis for HNC prognosis estimation and treatment planning.

The identification of disease-associated modules via co-expression analysis has emerged as a powerful method of obtaining novel insights into cancer biology (33). A number of studies have identified that gene signatures may predict the early detection, clinical stage, survival outcome or treatment of cancer (34-36). Based on WGCNA, Yuan et al (15) reported that COL3A1 was associated with the aggressiveness and poor prognosis of breast cancer with the possible mechanism of regulating the MAPK pathway. Zhou et al (16) reported that ten hub genes (cyclin B1, centromere protein F, DLG associated protein 5, cyclin A2, kinesin family member 14, NIMA related kinase 2 , kinesin family member 23 , TPX2, ubiquitin conjugating enzyme E2C and Rac GTPase activating protein 1), which were associated with tumor progression and prognosis, were identified in pancreatic carcinoma. Liu et al (17) identified essential genes involved in the pathogenesis of osteosarcoma by constructing a gene co-expression network. Using the WGCNA approach, the blue and black modules associated 

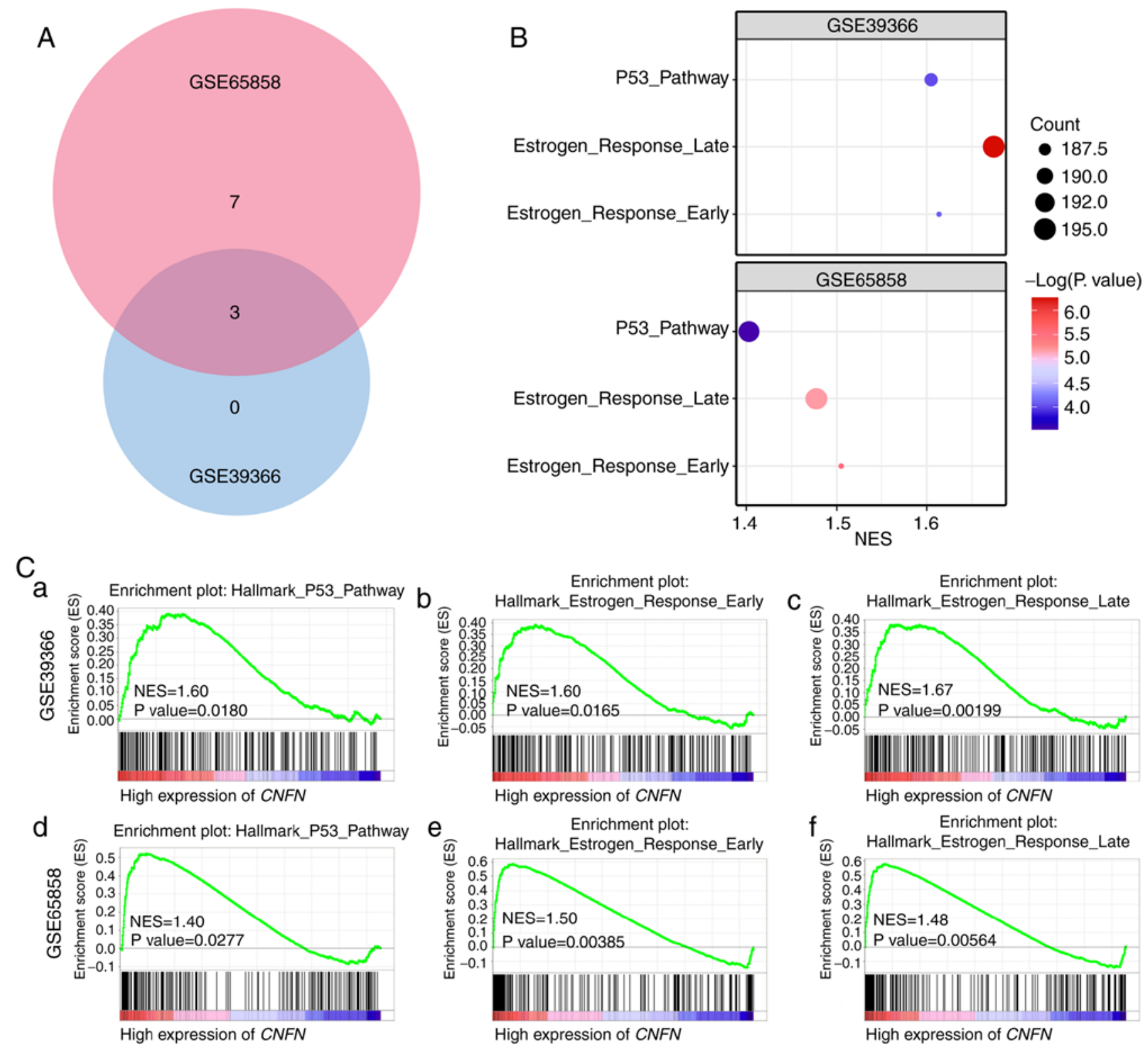

Figure 9. GSEA analysis. (A) Venn plot of the GSEA results of the GSE65858 and The Cancer Genome Atlas head and neck squamous cell carcinoma datasets. (B) Bubble plot of the significant gene sets. (Ca-f) Significant gene set. GSEA, gene set enrichment analysis; CNFN, cornifelin; P53, cellular tumor antigen P53.

with the $\mathrm{N}$ stage of $\mathrm{HNC}$ were screened in the present study. A total of 18 genes with high connectivity in the two modules were distinguished as candidate hub genes. These genes were enriched in the 'P53,' 'estrogen response early,' and 'estrogen response late' pathways via pathway enrichment analysis of GSEA. Following verification, CNFN and DSG1 were obtained as real hub genes closely associated with the $\mathrm{N}$ stage of $\mathrm{HNC}$ and vital biological processes. Following further validation via survival analysis, CNFN was demonstrated to be more tightly correlated with survival compared with DSG1.

CNFN (cornifelin, also termed PLAC8L2) is highly expressed in the esophagus and skin, and is located on chromosome 19q13.2. There are few reports on CNFN, and no reports verifying its function, to the best of our knowledge. Huang et al (37) revealed that CNFN was one of the core genes in the placental tissue involved in the development of gestational diabetes mellitus. Michibata et al (38) demonstrated that CNFN had increased expression in psoriatic skin. As one of the novel UVB signature genes, CNFN may be utilized to predict UVB photobiological effects on the skin and skin carcinogenesis (39). Zhang et al (40) reported that CNFN was potentially important for breast cancer due to its differential expression in tumors compared with normal breast tissues. Excluding these reports, no research on CNFN and other diseases was identified. In the present study, CNFN was regarded as the key hub gene associated with the clinical stage of HNC with survival differences, and exhibited differential expression between normal and tumor samples of HNSC, suggesting that CNFN may be used as a biomarker for assigning the correct clinical stage and for estimating the prognosis of patients with HNC.

The functional and pathway enrichment analysis indicated that three gene sets, 'P53 pathway', 'estrogen response early' and 'estrogen response late', were significantly enriched. The P53 pathway, one of the canonical pathways controlling cell-cycle progression, cell growth and apoptosis, has been reported to serve important roles in natural malignancy 
carcinogenesis (41-43). TP53, also known as Tp53 or p53, is frequently altered in human cancer. The reactivation of p53 activity in tumors results in tumor suppression in vivo (44). Oncogenes are overexpressed in numerous cancer types, thereby inhibiting the expression of tumor suppressor p53 (45). Wade et al (46) reported that as an oncogene, MDMX is overexpressed in a number of tumors, including breast and colorectal cancer, melanoma and osteosarcoma, leading to the suppression of tumor suppressor p53. The amplification of MDMX may inhibit the anticancer effects of the p53 protein and lead to tumor resistance (45). Venkatanarayan et al (44) reported that pramlintide, a synthetic analog of amylin, was extremely effective for p53-deficient thymic lymphomas, indicating a novel therapeutic strategy to target p53-deficient tumors. Therefore, personalized cancer therapy that is based on targeting the P53 pathway is an appealing therapeutic strategy for treating cancer with P53 pathway dysfunction. The P53 pathway is frequently co-altered with other pathways. One alteration of this canonical pathway is sufficient to alter others functionally, and pathways frequently have multiple alterations in one tumor sample (43). For example, in the small intestine and colon, the suppression of APC produces adenomas; with mutations of Kras and p53, this may progress to invasive carcinoma (47). To maintain homeostasis and proper cellular function, large tumor suppressor kinases 1 and 2, the Dbf2-related kinases, have emerged as central regulators of cell fate by modulating the p53 and estrogen pathways (48). Kundu et al (49) indicated that in certain $\mathrm{ER}+$ breast cancers the estrogen-MDM2-Rb-E2F1 axis is a central hub for estrogen-mediated p53-independent signal transduction. Zwijsen et al (50) demonstrated that estrogen, which acts via binding to a specific estrogen receptor (ER), played an important role in regulating the cell proliferation of the female breast. In breast cancer, the estrogen response and ERBB2/HER-2 pathways have long been implicated in etiology and drug response (51). Hsu et al (52) reported that aberrantly amplified estrogen response elements may potentially deregulate target gene expression associated with breast cancer development. CNFN, the gene identified in the present study to be closely associated with $\mathrm{N}$ stage and survival, may be involved in the P53 pathway and estrogen response. Therefore, further exploration of the P53 pathway and estrogen response, in addition to the associated genes, is warranted.

Although the present study identified hub genes associated with lymph node metastasis and survival via bioinformatics methods, no experimental study of these real hub genes was conducted, which is a limitation of the study. A clinical study and a functional analysis in vivo and in vitro are required to investigate the functions of these genes further. In conclusion, CNFN was involved in the progression of lymph node metastasis in HNC. This correlation provided a hypothesis that genes associated with $\mathrm{N}$ stage may have an essential role in deciding $\mathrm{HNC}$ metastatic progression. To elucidate additional carcinogenesis and metastasis targets, more basic functional studies are required to investigate these selected genes further.

\section{Acknowledgements}

This study is a partial fulfillment of the requirements for the doctoral degrees of Dr Xingya Gao and Dr Xia He. The authors would like to thank the Jiangsu Cancer Hospital and No. 2 People's Hospital of Lianyungang for their assistance.

\section{Funding}

This study was supported by the Young and Middle-aged Medical Talent Growth Fund of No. 2 People's Hospital of Lianyungang (grant no. TQ201701), the National Natural Science Foundation of China (grant no. 81672989), the National Natural Science Foundation of China (grant no. 81702685), and the fifth phase of the ' 333 Project' medium of Jiangsu Province (grant no. BRA2016523).

\section{Availability of data and materials}

All data generated or analyzed during this study are included in this published article.

\section{Authors' contributions}

$\mathrm{BL}, \mathrm{LY}, \mathrm{XG}$ and $\mathrm{XH}$ are guarantors of the integrity of the entire study. BL, LY, XG and XH conceived the study. BL, $\mathrm{XG}$ and $\mathrm{XH}$ designed the study. GH, XT and HZ performed data acquisition. BL, ZM and GH analyzed and interpreted the data. BL performed the statistical analysis and wrote the manuscript. XT and LY were responsible for manuscript revision/review. All authors read and approved the manuscript and agree to be accountable for all aspects of the research in ensuring that the accuracy or integrity of any part of the work are appropriately investigated and resolved.

\section{Ethics approval and consent to participate}

Not applicable.

\section{Patient consent for publication}

Not applicable.

\section{Competing interests}

The authors declare that they have no competing interests.

\section{References}

1. Leemans CR, Snijders PJF and Brakenhoff RH: The molecular landscape of head and neck cancer. Nat Rev Cancer 18: 269-282, 2018.

2. Shield KD,Ferlay J, Jemal A, Sankaranarayanan R, Chaturvedi AK, Bray F and Soerjomataram I: The global incidence of lip, oral cavity, and pharyngeal cancers by subsite in 2012. CA Cancer J Clin 67: 51-64, 2017.

3. Denaro N, Russi EG and Merlano MC: Pros and cons of the new edition of TNM classification of head and neck squamous cell carcinoma. Oncology 95: 202-210, 2018.

4. Chi AC, Day TA and Neville BW: Oral cavity and oropharyngeal squamous cell carcinoma-An update. CA Cancer J Clin 65: 401-421, 2015.

5. Steuer CE, El-Deiry M, Parks JR, Higgins KA and Saba NF: An update on larynx cancer. CA Cancer J Clin 67: 31-50, 2017.

6. Pereira ER, Kedrin D, Seano G, Gautier O, Meijer EFJ, Jones D, Chin SM, Kitahara S, Bouta EM, Chang J, et al: Lymph node metastases can invade local blood vessels, exit the node, and colonize distant organs in mice. Science 359: 1403-1407, 2018. 
7. Ozsolak F, Platt AR, Jones DR, Reifenberger JG, Sass LE, McInerney P, Thompson JF, Bowers J, Jarosz M and Milos PM: Direct RNA sequencing. Nature 461: 814-818, 2009.

8. Keck MK, Zuo Z, Khattri A, Stricker TP, Brown CD, Imanguli M, Rieke D, Endhardt K, Fang P, Brägelmann J, et al: Integrative analysis of head and neck cancer identifies two biologically distinct HPV and three non-HPV subtypes. Clin Cancer Res 21 $870-881,2015$

9. Walter V, Yin X, Wilkerson MD, Cabanski CR, Zhao N, Du Y, Ang MK, Hayward MC, Salazar AH, Hoadley KA, et al: Molecular subtypes in head and neck cancer exhibit distinct patterns of chromosomal gain and loss of canonical cancer genes. PLoS One 8: e56823, 2013.

10. Peri S, Izumchenko E, Schubert AD, Slifker MJ, Ruth K, Serebriiskii IG, Guo T, Burtness BA, Mehra R, Ross EA, et al: NSD1- and NSD2-damaging mutations define a subset of laryngeal tumors with favorable prognosis. Nat Commun 8: 1772, 2017.

11. Tartour E, Mosseri V, Jouffroy T, Deneux L, Jaulerry C, Brunin F, Fridman WH and Rodriguez J: Serum soluble interleukin-2 receptor concentrations as an independent prognostic marker in head and neck cancer. Lancet 357: 1263-1264, 2001.

12. Lin MC, Chien PH, Wu HY, Chen ST, Juan HF, Lou PJ and Huang MC: C1GALT1 predicts poor prognosis and is a potential therapeutic target in head and neck cancer. Oncogene 37 5780-5793, 2018.

13. Rettig EM, Bishop JA, Agrawal N, Chung CH, Sharma R, Zamuner F, Li RJ, Koch WM, Califano JA, Guo T, et al: HEY1 is expressed independent of NOTCH1 and is associated with poor prognosis in head and neck squamous cell carcinoma. Oral Oncol 82: 168-175, 2018.

14. Langfelder $P$ and Horvath S: WGCNA: An R package for weighted correlation network analysis. BMC Bioinformatics 9: 559, 2008.

15. Yuan L, Shu B, Chen L, Qian K, Wang Y, Qian G, Zhu Y, Cao X, Xie C, Xiao Y and Wang X: Overexpression of COL3A1 confers a poor prognosis in human bladder cancer identified by co-expression analysis. Oncotarget 8: 70508-70520, 2017.

16. Zhou Z, Cheng Y, Jiang Y, Liu S, Zhang M, Liu J and Zhao Q: Ten hub genes associated with progression and prognosis of pancreatic carcinoma identified by co-expression analysis. Int J Biol Sci 14: 124-136, 2018.

17. Liu X, Hu AX, Zhao JL and Chen FL: Identification of key gene modules in human osteosarcoma by co-expression analysis weighted gene co-expression network analysis (WGCNA). J Cell Biochem 118: 3953-3959, 2017

18. Zhang X, Feng H, Li Z, Li D, Liu S, Huang H and Li M: Application of weighted gene co-expression network analysis to identify key modules and hub genes in oral squamous cell carcinoma tumorigenesis. Onco Targets Ther 11: 6001-6021, 2018.

19. Li XT: Identification of key genes for laryngeal squamous cell carcinoma using weighted co-expression network analysis. Oncol Lett 11: 3327-3331, 2016.

20. Wichmann G, Rosolowski M, Krohn K, Kreuz M, Boehm A Reiche A, Scharrer U, Halama D, Bertolini J, Bauer U, et al: The role of HPV RNA transcription, immune response-related gene expression and disruptive TP53 mutations in diagnostic and prognostic profiling of head and neck cancer. Int J Cancer 137: 2846-2857, 2015.

21. Gautier L, Cope L, Bolstad BM and Irizarry RA: Affy-analysis of affymetrix genechip data at the probe level. Bioinformatics 20 307-315, 2004 .

22. Zhou Z, Liu S, Zhang M, Zhou R, Liu J, Chang Y and Zhao Q: Overexpression of topoisomerase 2-alpha confers a poor prognosis in pancreatic adenocarcinoma identified by co-expression analysis. Dig Dis Sci 62: 2790-2800, 2017.

23. Mason MJ, Fan G, Plath K, Zhou Q and Horvath S: Signed weighted gene co-expression network analysis of transcriptional regulation in murine embryonic stem cells. BMC Genomics 10 $327,2009$.

24. Yuan L, Chen L, Qian K, Wang G, Lu M, Qian G, Cao X, Jiang W, Xiao Y and Wang X: A novel correlation between ATP5A1 gene expression and progression of human clear cell renal cell carcinoma identified by co-expression analysis. Oncol Rep 39: 525-536, 2018.

25. Botia JA, Vandrovcova J, Forabosco P, Guelfi S, D'Sa K, United Kingdom Brain Expression Consortium, Hardy J, Lewis CM, Ryten M and Weale ME: An additional k-means clustering step improves the biological features of WGCNA gene co-expression networks. BMC Syst Biol 11: 47, 2017.
26. Foroushani A, Agrahari R, Docking R, Chang L, Duns G, Hudoba M, Karsan A and Zare H: Large-scale gene network analysis reveals the significance of extracellular matrix pathway and homeobox genes in acute myeloid leukemia: An introduction to the Pigengene package and its applications. BMC Med Genomics 10: 16, 2017.

27. Forabosco P, Ramasamy A, Trabzuni D, Walker R, Smith C, Bras J, Levine AP, Hardy J, Pocock JM, Guerreiro R, et al: Insights into TREM2 biology by network analysis of human brain gene expression data. Neurobiol Aging 34: 2699-2714, 2013.

28. Subramanian A, Kuehn H, Gould J, Tamayo P and Mesirov JP: GSEA-P: A desktop application for gene set enrichment analysis. Bioinformatics 23: 3251-3253, 2007.

29. Lydiatt WM, Patel SG, O'Sullivan B, Brandwein MS, Ridge JA, Migliacci JC, Loomis AM and Shah JP: Head and neck cancers-major changes in the American joint committee on cancer eighth edition cancer staging manual. CA Cancer J Clin 67: 122-137, 2017.

30. Ferris RL, Lotze MT, Leong SP, Hoon DS and Morton DL: Lymphatics, lymph nodes and the immune system: Barriers and gateways for cancer spread. Clin Exp Metastasis 29: 729-736, 2012.

31. Kawada K and Taketo MM: Significance and mechanism of lymph node metastasis in cancer progression. Cancer Res 71: 1214-1218, 2011.

32. Saksena MA, Saokar A and Harisinghani MG: Lymphotropic nanoparticle enhanced MR imaging (LNMRI) technique for lymph node imaging. Eur J Radiol 58: 367-374, 2006.

33. Grobner SN, Worst BC, Weischenfeldt J, Buchhalter I, Kleinheinz K, Rudneva VA, Johann PD, Balasubramanian GP, Segura-Wang M, Brabetz S, et al: The landscape of genomic alterations across childhood cancers. Nature 555: 321-327, 2018.

34. Wang QL, Chen X, Zhang MH, Shen QH and Qin ZM: Identification of hub genes and pathways associated with retinoblastoma based on co-expression network analysis. Genet Mol Res 14: 16151-16161, 2015.

35. Zhou R and Man Y: Integrated analysis of DNA methylation profiles and gene expression profiles to identify genes associated with pilocytic astrocytomas. Mol Med Rep 13: 3491-3497, 2016.

36. Liu J, Jing L and Tu X: Weighted gene co-expression network analysis identifies specific modules and hub genes related to coronary artery disease. BMC Cardiovasc Disord 16: 54, 2016.

37. Huang C, Huang BB, Niu JM, Yu Y, Qin XY, Yang YL, Xiao TX, Chen J, Ren LR and Zhang JV: Global mRNA and long non-coding RNA expression in the placenta and white adipose tissue of mice fed a high-fat diet during pregnancy. Cell Physiol Biochem 50: 2260-2271, 2018.

38. Michibata H, Chiba H, Wakimoto K, Seishima M, Kawasaki S, Okubo K, Mitsui H, Torii $\mathrm{H}$ and Imai Y: Identification and characterization of a novel component of the cornified envelope, cornifelin. Biochem Biophys Res Commun 318: 803-813, 2004.

39. Sun X, Kim A, Nakatani M, Shen Y and Liu L: Distinctive molecular responses to ultraviolet radiation between keratinocytes and melanocytes. Exp Dermatol 25: 708-713, 2016.

40. Zhang B, Chen MY, Shen YJ, Zhuo XB, Gao P, Zhou FS, Liang B, $\mathrm{Zu}$ J, Zhang $\mathrm{Q}$ and Suleman S: A large-scale, exome-wide association study of han chinese women identifies three novel loci predisposing to breast cancer. Cancer Res 78: 3087-3097, 2018.

41. Till JE, Yoon C, Kim BJ, Roby K, Addai P, Jonokuchi E, Tang LH, Yoon SS and Ryeom S: Oncogenic KRAS and p53 loss drive gastric tumorigenesis in mice that can be attenuated by E-Cadherin expression. Cancer Res 77: 5349-5359, 2017.

42. Turrell FK, Kerr EM, Gao M, Thorpe H, Doherty GJ, Cridge J, Shorthouse D, Speed A, Samarajiwa S, Hall BA, et al: Lung tumors with distinct p53 mutations respond similarly to p53 targeted therapy but exhibit genotype-specific statin sensitivity. Genes Dev 31: 1339-1353, 2017

43. Sanchez-Vega F, Mina M, Armenia J, Chatila WK, Luna A, La KC, Dimitriadoy S, Liu DL, Kantheti HS, Saghafinia S, et al: Oncogenic signaling pathways in the cancer genome atlas. Cell 173: 321-337 2018.

44. Venkatanarayan A, Raulji P, Norton W, Chakravarti D, Coarfa C, Su X, Sandur SK, Ramirez MS, Lee J, Kingsley CV, et al: IAPP-driven metabolic reprogramming induces regression of p53-deficient tumours in vivo. Nature 517: 626-630, 2015.

45. Chen SH, Forrester W and Lahav G: Schedule-dependent interaction between anticancer treatments. Science 351: 1204-1208, 2016. 
46. Wade M, Li YC and Wahl GM: MDM2, MDMX and p53 in oncogenesis and cancer therapy. Nat Rev Cancer 13: 83-96, 2013.

47. Dow LE, O'Rourke KP, Simon J, Tschaharganeh DF, van Es JH, Clevers $\mathrm{H}$ and Lowe SW: Apc restoration promotes cellular differentiation and reestablishes crypt homeostasis in colorectal cancer. Cell 161: 1539-1552, 2015.

48. Furth N and Aylon Y: The LATS1 and LATS2 tumor suppressors: Beyond the Hippo pathway. Cell Death Differ 24: 1488-1501, 2017.

49. Kundu N, Brekman A, Kim JY, Xiao G, Gao C and Bargonetti J: Estrogen-activated MDM2 disrupts mammary tissue architecture through a p53-independent pathway. Oncotarget 8: 47916-47930, 2017.

50. Zwijsen RM, Wientjens E, Klompmaker R, van der Sman J, Bernards R and Michalides RJ: CDK-independent activation of estrogen receptor by cyclin D1. Cell 88: 405-415, 1997.
51. Hurtado A, Holmes KA, Geistlinger TR, Hutcheson IR, Nicholson RI, Brown M, Jiang J, Howat WJ, Ali S and Carroll JS: Regulation of ERBB2 by oestrogen receptor-PAX2 determines response to tamoxifen. Nature 456: 663-666, 2008.

52. Hsu PY, Hsu HK, Lan X, Juan L, Yan PS, Labanowska J, Heerema N, Hsiao TH, Chiu YC, Chen Y, et al: Amplification of distant estrogen response elements deregulates target genes associated with tamoxifen resistance in breast cancer. Cancer Cell 24: 197-212, 2013.

(7) (9) This work is licensed under a Creative Commons Attribution-NonCommercial-NoDerivatives 4.0 International (CC BY-NC-ND 4.0) License. 